\title{
A hybrid method for decision making with dependence \& feedback under incomplete information
}

\author{
Weijie Chen* \& Yan Zou \\ School of Economics and Management, Chongqing Normal University, Chongqing, China
}

\begin{abstract}
This paper presents a hybrid method to tackle multiple criteria decision making problems with incomplete weight information in the context of fuzzy soft sets. In order to determine the weights of criteria, we develop a comprehensive two-stage framework. Stage One: We first define the distance between two fuzzy soft numbers. Next, we establish an optimization model based on ideal point of attribute values, by which the attribute weights can be determined. Stage Two: To get the global weights, we use fuzzy cognitive maps to depict the dependent and feedback effect among criteria. Next, we require constructing fuzzy soft set to decide the desirable alternative. Finally, a case study is given to clarify the proposed approach of this paper.
\end{abstract}

Keywords: MCDM; Fuzzy soft set; FCM

\section{INTRODUCTION}

Multi-criteria decision making (MCDM) refers to making preference decisions over the available alternatives that are characterized by multiple, usually conflicting attributes [1]. It occurs in a variety of actual situations, such as economic analysis, strategic planning, forecasting, medical diagnosis, supply chain management and many other areas. Due to the increasing complexity of the socio-economic environment, this has made it even more difficult for decision making, which is mainly shown in two aspects.

On the one hand, due to the complexity and the situation of uncertainty in decision, the information about attribute weights provided by decision makers is usually incompletely known. Some of recent research on the topic incorporates generalized interval-valued fuzzy numbers [2], triangular fuzzy number [3], intuitionistic fuzzy set [4, 5], 2-tuple linguistic [6] and others. Although these literatures are very good for solving the incomplete weight information under uncertain environment, they are associated with an inherent limitation, which is inadequacy of the parameterization tool associated with these theories. Yet, soft set which was initiated by [7], a new mathematical tool can deal with uncertainties, which is free from the above limitations.

In recent years, the research on soft set theory has

*Corresponding author: chwj721@163.com achieved great progress in theoretical aspect. At the same time, there has been some progress concerning practical applications of soft set theory, especially the use of soft sets in decision making. Maji and Roy [8] introduced the definition of reduct-soft-set and described the application of soft set theory as a problem in decision-making. Mushrif et al. [9] proposed a new classification algorithm of the natural textures, which was based on the notions of soft set theory. Zou and Xiao [10] presented data analysis approaches of soft set under incomplete information. Roy and Maji [11] proposed a novel method of object recognition from an imprecise multi-observer data and a decision making application of fuzzy soft set. Although the algorithm was proved incorrectly by Kong et al. [12], fuzzy soft sets and multi-observer concepts are valuable to successive researchers. Cagman and Enginoglu [13] defined products of soft sets and uni-int decision function. By using these new definitions, they constructed a uni-int decision making method which selected a set of optimum elements from the alternatives. Feng et al. [14] presented an adjustable approach to fuzzy soft set based decision making and enhanced it with illustrations. Although fuzzy soft set has been progressive in decision making, few literatures concentrated on the decision making with incomplete weight information. Therefore, we constructed a decision model based on fuzzy soft set to determine unknown weight in this paper. 
On the other hand, MCDM is also involved in determining the optimal alternative among multiple, conflicting and interactive criteria [15]. For most previous literature, they assumed that attributes were mutually independent. However, in real-life situation there exist dependence and feedback effects simultaneously among criteria, while making decisions. The analytic network process (ANP) which was proposed in $[16,17]$, overcomes the problem of dependence and feedback effect among criteria or alternatives. Though ANP has been widely used in various applications, there are still two main problems which were highlighted by [18]. To deal with this problem, we use FCM to express dependence and feedback effect among criteria in order to overcome the preferential dependent and shortcomings of ANP.

Based on above analysis, it is hard for decision makers to make a good decision using a simple weighted method because of attribute weights with interaction effects and incomplete information. So it will be an interesting and important research topic as few literatures considered them simultaneously. To fill this gap, we presented a hybrid multi-criteria decision making approach based on fuzzy soft set and FCM. The proposed method not only enriches fuzzy soft set theory, but also expands the field of decision making.

As for the remainder of this paper, it is organized as follows: Section 2 will review some basic concepts related to fuzzy soft set and fuzzy cognitive maps. In Section 3, we will provide a new hybrid model to determine the attribute weights considering the incomplete information, the independent and feedback effect among criteria. A fuzzy soft set will also be introduced for multiple attribute decision making problems. Next, a case study is developed to demonstrate on how to apply the proposed approach in Section 4. Finally, conclusion and remarks will be in Section 5.

\section{THEORETICAL BACKGROUND}

In this section, we will briefly review the basic theoretical background on fuzzy soft set and fuzzy cognitive maps.

\subsection{Fuzzy soft set}

Definition 2.1 [11]: Let $\mathscr{P}(U)$ be the set of all fuzzy subsets in an initial universe $U$. Let $E$ be a set of parameters and $A \subset E$. A pair $(\tilde{F}, A)$ is called a fuzzy soft set over $U$, where $\tilde{F}$ is a mapping given by

$$
\tilde{F}: A \rightarrow \mathscr{P}(U)
$$

Definition 2.2 [12]: Let $f_{i j}(i=1,2, \ldots n ; j=1,2, \ldots m . i, j$ denote row vector and column vector of the tabular representation for fuzzy soft set, respectively.) be the element of resultant fuzzy soft set. Then we call $C=\left(c_{i j}\right)_{\mathrm{n} \times \mathrm{m}}$ the score matrix of the resultant fuzzy soft set where $c_{i j}=\sum_{k=1}^{n}\left(f_{i j}-f_{k j}\right)$, and $c_{i}$ is a choice value for each alternative $h_{i}$, such that

$c_{i}=\sum_{j=1}^{m} c_{i j}$

Based on the choice value formula, we presented the overall choice values of each alternative $h_{j}(i=1,2, \ldots n)$ :

$c_{i}(w)=\sum_{j=1}^{m} w_{j} c_{i j}, i=1,2, \ldots, n$

\subsection{The basic theory of fuzzy cognitive maps}

FCM is a soft computing tool, which combines the elements of fuzzy logic and neural networks. Strictly speaking, fuzzy cognitive map is a directed cyclic graph composed by the nodes and edges. Nodes of the map are commonly known as the concepts which indicating the main features, nature or attributes of the system. The edges between nodes show the various causal relationships. Figure 1 illustrates a simple FCM, where each concept node $C_{i}$ in fuzzy cognitive map corresponds to a concept value $A_{i} \in[0,1]$, and the edges between the nodes correspond to a value, shows by the connection weight $w_{i j}$, in the interval $[-1,1]$. The weights correspond to the three main situations: positive, negative and zero, indicating that the concepts of positive correlation, negative correlation and not related respectively, and the absolute values reflect the extent of the impact between the concepts.

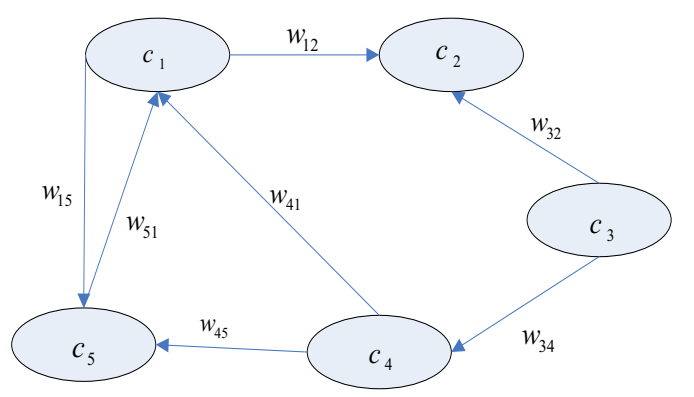

Figure 1. A simple fuzzy cognitive map

The values of the connection weights can be organized in a matrix,

$$
\begin{array}{c|cccc} 
& C_{1} & C_{2} & \cdots & C_{N} \\
C_{1} & w_{11} & w_{12} & \cdots & w_{1 N} \\
C_{2} & w_{21} & w_{22} & \cdots & w_{2 N} \\
\vdots & \vdots & \vdots & \ddots & \vdots \\
C_{N} & w_{N 1} & w_{N 2} & \cdots & w_{N N}
\end{array} \mid
$$

The reasoning process of FCM can be expressed as 
[19]:

$$
A_{i}^{(k+1)}=f\left(A_{i}^{(k)}+\sum_{j=1, j \neq i}^{N} A_{j}^{(k)} w_{j i}\right)
$$

Among them, $A_{i}^{(k+1)}$ is the value of concept $C_{i}$ at simulation step $k+1 ; A_{j}^{(k)}$ is the value of concept $C_{j}$ at simulation step $k ; w_{i j}$ is the weight of the interconnection between concept $C_{j}$ and $C_{i} ; f$ is a threshold function, which is used to ensure the node concept value in the interval $[0,1]$. The threshold function $f$ can be bivalent $(f(x)=0$ or 1$)$, trivalent $(f(x)=-1,0$ or 1$)$, tangent hyperbolic $(f(x)=\tanh (x))$ or the unipolar sigmoid function $\left(f(x)=1 /\left(1+e^{-c x}\right)\right)$, where $c>0$ determines the steepness of the continuous function $f$. The sigmoid function is typically used when the concept interval is $[0,1]$. Hyperbolic function is used when concepts can be negative and their values belong to the interval $[-1,1]$.Thus the selection of threshold function depends on the description of concepts.

If the reasoning process achieves one of the following three states, one has reached a steady state, and ends the iteration: output concept value has stabilized at a fixed value; changes of the values have shown signs of cyclical; chaotic state has appeared, that is, the concept value is uncertain and random.

\section{A HYBRID AND RATIONAL METHOD FOR MULTI-CRITERIA DECISION MAKING BASED ON FUZZY SOFT SET AND FCM}

This section presents a hybrid and rational approach to tackle multiple criteria decision making problems with incomplete weight information in the context of fuzzy soft sets. Suppose that there exist an alternative set $U=\left\{h_{1}, h_{2}, \ldots, h_{n}\right\}$, consisting of $n$ non-inferior alternatives, and an attribute set $E=\left\{e_{1}, e_{2}, \ldots, e_{m}\right\}$. Each alternative is assessed on the $m$ attributes. The decision problem is to select a most preferred alternative from set $U$ based on the overall assessments of all alternatives on the $m$ attributes. Due to the complexity and the situation of uncertainty in decision, the information about attribute weights provided by the decision makers is usually incompletely known and the dependent and feedback effect among criteria cannot be ignored.

In the following, we proposed a hybrid and rational method that offers a possibility for handing these issues simultaneously. The framework of decision making can be shown in Figure 2.

\subsection{A rational model for determining initial criteria weight}

In this section, we will discuss all kinds of optimization models considering decision makers' rationality to determine the weights of attributes.

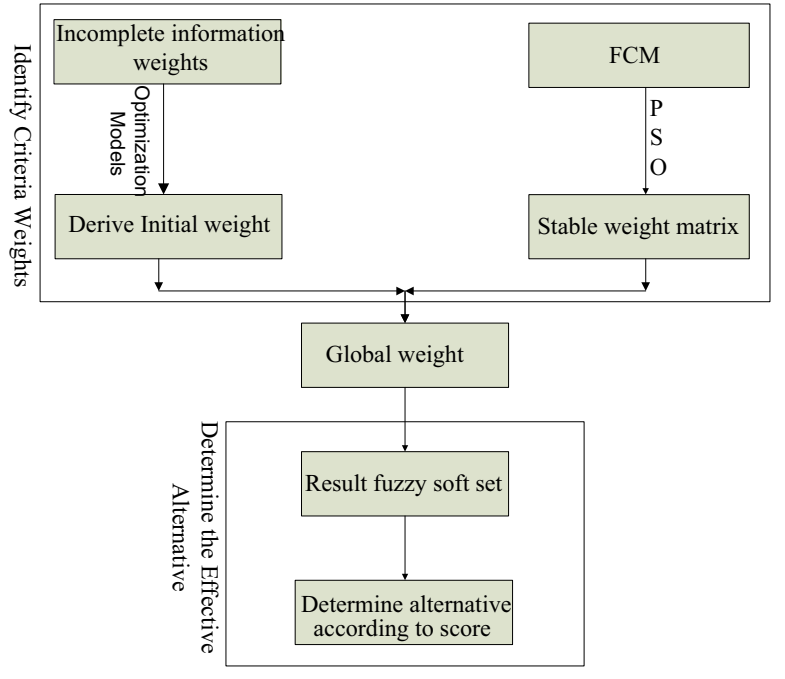

Figure 2 Stepwise procedure

Definition 3.1: Let $(\tilde{F}, A)$ be a fuzzy soft set, and then the deviation from the PIS for any element $\tilde{F}(\varepsilon)\left(h_{k}\right) \quad(k=1,2, \ldots, n)$ can be defined as follows:

$\tilde{d}\left(\tilde{F}(\varepsilon)\left(h_{k}\right), \tilde{F}(\varepsilon)\left(h_{p}\right)\right)=\left|\tilde{F}(\varepsilon)\left(h_{k}\right)-\tilde{F}(\varepsilon)\left(h_{p}\right)\right|$

Wher $\tilde{F}(\varepsilon)\left(h_{k}\right)(k=1,2, \ldots, n)$ represents $\varepsilon$-approximate for alternative $h_{\mathrm{k}}$ of the fuzzy soft set and $\tilde{F}(\varepsilon)\left(h_{p}\right)$ represents PIS for $\varepsilon$-approximate of the fuzzy soft set. So we can also define the distance of two fuzzy soft set.

$\tilde{d}\left((\tilde{F}, w),\left(\tilde{F}_{i}, w\right)\right)=\sum_{j=1}^{m} w_{j} \tilde{d}\left(\tilde{F}\left(\varepsilon_{j}\right)\left(h_{i j}\right), \tilde{F}\left(\varepsilon_{j}\right)\left(h_{p j}\right)\right)$

Where $\tilde{d}\left((\tilde{F}, w),\left(\tilde{F}_{i}, w\right)\right)$ denotes the distance between the fuzzy soft set $(F, w)$ of ideal point and the fuzzy soft set $\left(\tilde{F}_{i}, w\right)$ of the alternative $h_{i}$ :

Obviously, the smaller $\tilde{d}\left((\tilde{F}, w),\left(\tilde{F}_{i}, w\right)\right)$, the better the alternative $h_{i}$ will be.

Case 1: The information about attribute weights is incompletely known

1) The decision maker is completely optimistic

Due to the complexity and uncertainty of decision situation, the information about attribute weights provided by decision makers is usually incompletely known. Under [20] and [21] inspiration, we establish the following optimization model to minimize $\tilde{d}_{i}^{+}(w)$ :

(M-1) Minimize: $\tilde{d}_{i}^{+}(w)=\sum_{j=1}^{m} w_{j} \tilde{d}^{+}\left(\tilde{F}\left(\varepsilon_{j}\right)\left(h_{i j}\right), \tilde{F}\left(\varepsilon_{j}\right)\left(h_{p j}\right)\right)$

Subject to:

$$
w=\left(w_{1}, w_{2}, \ldots, w_{m}\right)^{T} \in H, w_{j} \geq 0, i=1,2, \ldots, m, \sum_{j=1}^{m} w_{j}=1 .
$$

Here $H$ is a set of constraints concerning the unknown weight information. For the sake of simplicity, they can only take the following forms, for $i \neq j$ : 
Form 1: A weak ranking: $\left\{w_{j 1} \geq w_{j 2}\right\}, j_{1} \neq j_{2}$;

Form 2: A strict ranking:

$\left\{w_{j_{1}}-w_{j_{2}} \geq \varepsilon_{j_{1} j_{2}}\right\}, j_{1} \neq j_{2}, \varepsilon_{j_{1} j_{2}}>0$;

Form 3: A ranking with multiples:

$\left\{w_{j_{1}} \geq \alpha_{j_{1} j_{2}} w_{j_{2}}\right\}, 0 \leq \alpha_{j_{1} j_{2}} \leq 1, j_{1} \neq j_{2}$;

Form 4: An interval form:

$\left\{\beta_{j} \leq w_{j} \leq \beta_{j}+\varepsilon_{j}\right\}, 0 \leq \beta_{j}<\beta_{j}+\varepsilon_{j} \leq 1 ;$

Form 5: A ranking of differences:

$\left\{w_{j 1} w_{j 2} \geq w_{j 2}\right\}\left\{w_{j_{1}}-w_{j_{2}} \geq w_{j_{3}}-w_{j_{4}}\right\}$, for $j_{1} \neq j_{2} \neq j_{3} \neq j_{4}$

Based on the above optimization model, we can see that the derived weight vector is overestimated because every alternative is often given its most ideal weighted condition.

2) If the decision maker is completely pessimistic, we used optimization model which minimized the deviation from NIS for all alternative. We establish the following optimization model to minimize $\tilde{d}_{i}^{-}(w)$ :

(M-2) Minimize: $\tilde{d}_{i}^{-}(w)=\sum_{j=1}^{m} w_{j} \tilde{d}^{-}\left(\tilde{F}\left(\varepsilon_{j}\right)\left(h_{i j}\right), \tilde{F}\left(\varepsilon_{j}\right)\left(h_{n j}\right)\right)$ Subject to:

$w=\left(w_{1}, w_{2}, \ldots, w_{m}\right)^{T} \in H, w_{j} \geq 0, i=1,2, \ldots, m, \sum_{j=1}^{m} w_{j}=1$.

Clearly in this model, the derived weight vector is too low estimated because every alternative is often given smallest ideal weighted condition.

So we can establish the optimization model:

\section{(M-3) Minimize}

$\tilde{d}_{i}^{+}(w)+\tilde{d}_{i}^{-}(w)=\sum_{j=1}^{m} w_{j}\left(\tilde{d}_{i}^{+}\left(\tilde{F}\left(\varepsilon_{j}\right)\left(h_{i j}\right), \tilde{F}\left(\varepsilon_{j}\right)\left(h_{p j}\right)\right)+\tilde{d}_{i}^{-}\left(\tilde{F}\left(\varepsilon_{j}\right)\left(h_{i j}\right), \tilde{F}\left(\varepsilon_{j}\right)\left(h_{n j}\right)\right)\right)$

Subject to:

$w=\left(w_{1}, w_{2}, \ldots, w_{m}\right)^{T} \in H, w_{j} \geq 0, i=1,2, \ldots, m, \sum_{j=1}^{m} w_{j}=1$.

By solving Model (M-3), we obtained the optimal solution $w^{(i)}=\left(w_{1}^{(i)}, w_{2}^{(i)}, \ldots, w_{m}^{(i)}\right)^{T}$ corresponding to the alternative $h_{i}$. However, in the process of determining the weight vector $w=\left(w_{1}, w_{2}, \ldots, w_{m}\right)^{T}$, we need to consider all the alternatives $h_{i}(i=1,2, \ldots, n)$ as a whole. Thus, we construct weight matrix $w=\left(w_{j}^{(i)}\right)_{m \times n}$ of the optimal solutions $w^{(i)}=\left(w_{1}^{(i)}, w_{2}^{(i)}, \ldots, w_{m}^{(i)}\right)^{T}$ $(i=1,2, \ldots n)$ as:

$w=\left(\begin{array}{cccc}w_{1}^{(1)} & w_{1}^{(2)} & \ldots & w_{1}^{(n)} \\ w_{2}^{(1)} & w_{2}^{(2)} & \ldots & w_{2}^{(n)} \\ \vdots & \vdots & \vdots & \vdots \\ w_{m}^{(1)} & w_{m}^{(2)} & \ldots & w_{m}^{(n)}\end{array}\right)$

And we calculate the normalized eigenvector $\omega=\left(\omega_{1}, \omega_{2}, \ldots, \omega_{n}\right)^{T}$ of the matrix

$$
\left(\left(\tilde{d}_{i}^{+}(w)+\tilde{d}_{i}^{-}(w)\right) w\right)^{T}\left(\left(\tilde{d}_{i}^{+}(w)+\tilde{d}_{i}^{-}(w)\right) w\right) .
$$

Then we can construct a combined weight vector as follows: $w^{\text {initial }}=w \omega=\left(\begin{array}{cccc}w_{1}^{(1)} & w_{1}^{(2)} & \ldots & w_{1}^{(n)} \\ w_{2}^{(1)} & w_{2}^{(2)} & \ldots & w_{2}^{(n)} \\ \vdots & \vdots & \vdots & \vdots \\ w_{m}^{(1)} & w_{m}^{(2)} & \ldots & w_{m}^{(n)}\end{array}\right)\left(\begin{array}{c}\omega_{1} \\ \omega_{2} \\ \vdots \\ \omega_{n}\end{array}\right)$

$=\omega_{1} w^{(1)}+\omega_{2} w^{(2)}+\ldots+\omega_{n} w^{(n)}$

Thus we can derive the local weight vector $w^{\text {initial }}=\left(w_{1}, w_{2}, \ldots, w_{m}\right)^{T}$.

Case 2: Completely unknown for weight information

If the information about attribute weights is completely unknown, Eq. (6) is replaced with the following deviation function

$\tilde{d}\left((\tilde{F}, w),\left(\tilde{F}_{i}, w\right)\right)=\sum_{j=1}^{m} w_{j}^{2}\left(\tilde{d}^{+}\left(\tilde{F}\left(\varepsilon_{j}\right)\left(\hat{h}_{i j}\right), \tilde{F}\left(\varepsilon_{j}\right)\left(h_{p j}\right)\right)+\tilde{d}^{-}\left(\tilde{F}\left(\varepsilon_{j}\right)\left(h_{i j}\right), \tilde{F}\left(\varepsilon_{j}\right)\left(h_{n j}\right)\right)\right)^{2}$

Obviously, the smaller $\tilde{d}\left((\tilde{F}, w),\left(\tilde{F}_{i}, w\right)\right)$ is, the better the alternative $h_{i}$ will be. Thus, a reasonable weight vector $w^{*}=\left(w_{1}^{*}, w_{2}^{*}, \ldots, w_{m}^{*}\right)$ should be determined so as to make all the distances $\tilde{d}\left((\tilde{F}, w),\left(\tilde{F}_{i}, w\right)\right)(i=1,2, \ldots, n)$ as smaller as possible, which means to minimize the following distance vector:

(M-4) Minimize

$\tilde{d}(w)=\left(\tilde{d}\left((\tilde{F}, w),\left(\tilde{F}_{1}, w\right)\right), \tilde{d}\left((\tilde{F}, w),\left(\tilde{F}_{2}, w\right)\right), \ldots, \tilde{d}\left((\tilde{F}, w),\left(\tilde{F}_{n}, w\right)\right)\right)$

Subject to:

$\sum_{j=1}^{m} w_{j}=1, w_{j} \geq 0, j=1,2, \ldots, m$.

where

$\tilde{d}\left((\tilde{F}, w),\left(\tilde{F}_{i}, w\right)\right)=\sum_{j=1}^{m} w_{j}^{2}\left(\tilde{d}^{+}\left(\tilde{F}\left(\varepsilon_{j}\right)\left(h_{i j}\right), \tilde{F}\left(\varepsilon_{j}\right)\left(h_{p j}\right)\right)+\tilde{d}^{-}\left(\tilde{F}\left(\varepsilon_{j}\right)\left(h_{i j}\right), \tilde{F}\left(\varepsilon_{j}\right)\left(h_{n j}\right)\right)\right)^{2}$

By linear equal weighted summation method [22, 6], the Model (M-4) can be transformed into a single-objective programming model:

(M-5) Minimize $\tilde{d}(w)=\sum_{i=1}^{n} \tilde{d}\left((\tilde{F}, w),\left(\tilde{F}_{i}, w\right)\right)$

Subject to: $\quad \sum_{j=1}^{m} w_{j}=1, w_{j} \geq 0, j=1,2, \ldots, m$

To solve this model, we constructed the Lagrange function:

$L(w, \lambda)=\tilde{d}(w)+2 \lambda\left(\sum_{j=1}^{m} w_{j}-1\right)$

Where $\lambda$ is the Lagrange multiplier.

Differentiating Eq. (8) with respect to $w_{j}(j=1,2, \ldots, m)$ and $\lambda$, and setting these partial derivatives equal to zero, the following set of equations is obtained:

$\left\{\begin{array}{c}\frac{\partial L(w, \lambda)}{\partial w_{j}}=2 \sum_{i=1}^{n}\left(\tilde{d}^{+}\left(\tilde{F}\left(\varepsilon_{j}\right)\left(h_{i j}\right), \tilde{F}\left(\varepsilon_{j}\right)\left(h_{p j}\right)\right)+\tilde{d}^{-}\left(\tilde{F}\left(\varepsilon_{j}\right)\left(h_{i j}\right), \tilde{F}\left(\varepsilon_{j}\right)\left(h_{h_{j}}\right)\right)\right)^{2} w_{j}+2 \lambda=0 \\ \frac{\partial L(w, \lambda)}{\partial \lambda}=\sum_{j=1}^{m} w_{j}-1=0\end{array}\right.$

By solving Eq. (9), we get a simple and exact formula for determining the attribute weights as follows: 


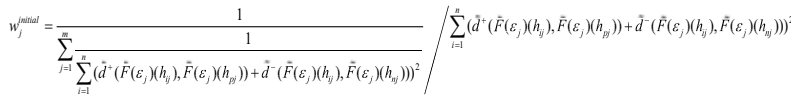

$$
\begin{aligned}
& (i=1,2, \ldots n ; j=1,2, \ldots, m)
\end{aligned}
$$

Which can be used as the weight vector of attributes, Obviously, $w_{j}^{\text {initial }} \geq 0$, for all $j$.

\subsection{A method for determining criteria global weight}

In this section, we will determine the criteria global weight. The procedure is shown in the following steps:

Step 1: Depict the fuzzy cognitive maps to indicate the influence among criteria by the experts and determine the connection matrix $W$ on the basis of FCM.

Step 2: Learn the connection matrix $W$ using PSO (particle swarm optimization) algorithm and calculate Eq. (4) to obtain the steady-state matrix $W^{*}$.

Step 3: Derive the global weight vector. In order to derive the global weights, we should first normalized the local weight vector $\left(w^{\text {initial }}\right)$ and the steady-state matrix $\left(W^{*}\right)$ as follows [18]:

$$
w_{n}^{\text {initial }}=\frac{1}{\lambda} w^{\text {initial }}
$$

and

$W_{n}^{*}=\frac{1}{\gamma} W^{*}$

where $\lambda$ is the largest element of $w^{\text {initial }}$ and $\gamma$ is the largest row sum of $W^{*}$. Then, we can obtain the global weight vector by using the following weighting equation [20]:

$w^{\text {global }}=w_{n}^{\text {initial }}+W_{n}^{*} w_{n}^{\text {initial }}$

\subsection{Evaluation and selection of alternatives}

The evaluation and selection of alternative is conducted in this section. Here we applied fuzzy soft set, which is a novel mathematical tool for dealing uncertainty, to evaluate alternative. The detailed procedures are as follows:

Step 1: Construct the resultant weighted fuzzy soft set $\left(\tilde{F},\left(w^{\text {global }} E\right)\right)$ according to the fuzzy soft set $(\tilde{F}, E) \cdot$

Step 2: According to the Eq. (3), we can calculate the comprehensive relative score of $h_{i}$, $\forall i, c_{i}\left(w^{\text {global }}\right)=\sum_{j=1}^{m} c_{i j}\left(w^{\text {global }}\right)$. The decision is $h_{i}$, if $c_{k}\left(w^{\text {global }}\right)=\max c_{i}\left(w^{\text {global }}\right)$.

\section{A CASE STUDY FOR EVALUATING SUPPLI- ER SELECTION PROBLEM}

In this section, we illustrate this hybrid evaluation and selection process by using a case study (adapted from [23]) as an example. A high-tech company which manufactures electronic products intends to evaluate and select a supplier of USB connectors from four suppliers $\left(h_{1}, h_{2}, h_{3}, h_{4}\right)$ conforming to the basic conditions of choice. In order to select the most suitable candidate, the decision makers take four attributes into account. They are the strong ability of delivery management $e_{1}$, the high integrated service capability $e_{2}$, the high quality management capability $e_{3}$ and the low price $e_{4}$. Due to the information acquisition difficult, we can only get part of the attribute weight information and the known attribute weight information is $h=\left\{\sum_{i=1}^{4} w_{i}=1 ; w_{1} \geq w_{2} ; w_{2} \geq 0.1 ; 0.37 \geq w_{3} \geq 0.3 ; w_{3} \geq 2 w_{4} ; w_{4} \geq 0.15 ; w_{i} \geq 0\right\}$.

According to the market forecast, the attribute value of each alternative is expressed as fuzzy soft set and the decision making data are shown in Table 1 . The structure of this performance evaluation problem is shown in Figure.3. The best supplier will be chosen according to the above decision-making information.

Table 1. Tabular representation of fuzzy soft set $(\tilde{F}, E)$ in the application example.

\begin{tabular}{lllll}
\hline$U$ & $e_{1}$ & $e_{2}$ & $e_{3}$ & $e_{4}$ \\
\hline$h_{1}$ & 0.95 & 0.89 & 0.80 & 0.89 \\
$h_{2}$ & 0.88 & 0.90 & 0.80 & 0.80 \\
$h_{3}$ & 0.90 & 0.93 & 0.85 & 0.78 \\
$h_{4}$ & 0.85 & 0.95 & 0.90 & 0.85 \\
\hline
\end{tabular}

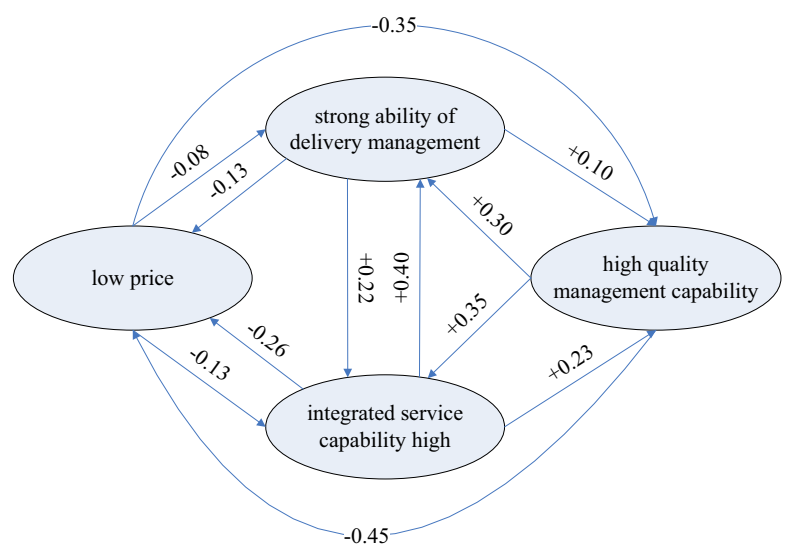

Figure 3. An fuzzy cognitive map

\subsection{The decision steps}

The proposed method is applied to solve this problem and the computational procedure is summarized as Figure 3.

Step 1: Identify the initial weights of attributes according to the incomplete weights information. 
Case 1: The information about attribute weights is incomplete known.

According to the characteristic of fuzzy soft set, we can define positive ideal solution and negative ideal solution of fuzzy soft set as follows:

positive ideal solution $_{(\tilde{F}, A)}=\left\{\left(e_{1}, 1\right),\left(e_{2}, 1\right), \ldots,\left(e_{m}, 1\right)\right\}$,

negative ideal solution $_{(\bar{F}, A)}=\left\{\left(e_{1}, 0\right),\left(e_{2}, 0\right), \ldots,\left(e_{m}, 0\right)\right\} \cdot$

Calculate the deviation from PIS and NIS for all the alternatives, which can be represented in the form of matrix $C_{p}$ and $C_{n}$, respectively.

$C_{p}=\left(\begin{array}{llll}0.05 & 0.11 & 0.20 & 0.11 \\ 0.12 & 0.10 & 0.20 & 0.20 \\ 0.10 & 0.07 & 0.15 & 0.22 \\ 0.15 & 0.05 & 0.10 & 0.15\end{array}\right), \quad C_{n}=\left(\begin{array}{cccc}0.95 & 0.89 & 0.80 & 0.89 \\ 0.88 & 0.90 & 0.80 & 0.80 \\ 0.90 & 0.93 & 0.85 & 0.78 \\ 0.85 & 0.95 & 0.90 & 0.85\end{array}\right)$.

Utilize the Model (M-1) to obtain the optimal weight vectors $w^{(j)}=\left(w_{1}^{(j)}, w_{2}^{(j)}, w_{3}^{(j)}, w_{4}^{(j)}\right)^{T}(j=1,2,3,4)$ corresponding to the alternatives $h_{j}(j=1,2,3,4)$ :

$w^{(1)}=(0.223,0.223,0.370,0.185)^{T}$

$w^{(2)}=(0.345,0.100,0.370,0.185)^{T}$

$w^{(3)}=(0.345,0.100,0.370,0.185)^{T}$

$w^{(4)}=(0.450,0.100,0.300,0.150)^{T}$

and construct the weight matrix:

$w=\left(\begin{array}{llll}0.223 & 0.345 & 0.345 & 0.450 \\ 0.223 & 0.100 & 0.100 & 0.100 \\ 0.370 & 0.370 & 0.370 & 0.300 \\ 0.185 & 0.185 & 0.185 & 0.150\end{array}\right)$

and then

$\left(C_{p} w\right)^{T}\left(C_{p} w\right)=\left(\begin{array}{llll}0.0725 & 0.0737 & 0.0737 & 0.0701 \\ 0.0737 & 0.0751 & 0.0751 & 0.0716 \\ 0.0737 & 0.0751 & 0.0751 & 0.0716 \\ 0.0701 & 0.0716 & 0.0716 & 0.0683\end{array}\right)$

Calculate the normalized eigenvectors $\omega$ of the matrix $\left(C_{p} w\right)^{T}\left(C_{p} w\right)$ :

$\omega=(0.2494,0.2542,0.2542,0.2422)^{T}$

Use Eq. (7) and derive the weight vector $w^{\text {initial }}$ :

$w^{\text {initial }}=w \omega=\left(\begin{array}{llll}0.223 & 0.345 & 0.345 & 0.450 \\ 0.223 & 0.100 & 0.100 & 0.100 \\ 0.370 & 0.370 & 0.370 & 0.300 \\ 0.185 & 0.185 & 0.185 & 0.150\end{array}\right)\left(\begin{array}{l}0.2494 \\ 0.2542 \\ 0.2542 \\ 0.2422\end{array}\right)$

$=(0.3400,0.1307,0.3530,0.1765)^{T}$

Step 2: According to the Figure 3, we can formulate the connection matrix $W$ as follows:

$W=\left(\begin{array}{llll}0.00 & 0.22 & 0.10 & 0.13 \\ 0.40 & 0.00 & 0.23 & 0.26 \\ 0.30 & 0.35 & 0.00 & 0.45 \\ 0.08 & 0.13 & 0.35 & 0.00\end{array}\right)$

Step 3: Using PSO learning method, we can obtain the steady-state matrix:

$W^{*}=\left(\begin{array}{cccc}0.000 & 0.300 & 0.050 & 0.100 \\ 0.330 & 0.000 & 0.250 & 0.200 \\ 0.200 & 0.400 & 0.000 & 0.350 \\ 0.056 & 0.150 & 0.300 & 0.000\end{array}\right)$

Step 4: According to Eq.(11),(12),(13), we can obtain the final global weights and calculate the normalized global weights:

$w^{\text {global }}=(0.25,0.23,0.32,0.20)^{T}$

Step 5: Construct the resultant weighted fuzzy soft set $\left(\tilde{F},\left(w^{\text {global }} E\right)\right)$ according to the fuzzy $\operatorname{soft} \operatorname{set}(\tilde{F}, E)$, which is shown in Table 2.

Table 2. Tabular representation of the resultant weighted fuzzy $\operatorname{soft} \operatorname{set}\left(\tilde{F},\left(w^{\text {global }} E\right)\right)$

\begin{tabular}{lllll}
\hline$U$ & $w_{1}^{\text {global }} e_{1}$ & $w_{2}^{\text {global }} e_{2}$ & $w_{3}^{\text {global }} e_{3}$ & $w_{4}^{\text {global }} e_{4}$ \\
\hline$h_{1}$ & 0.2375 & 0.2047 & 0.2560 & 0.1780 \\
$h_{2}$ & 0.2200 & 0.2070 & 0.2560 & 0.1600 \\
$h_{3}$ & 0.2250 & 0.2139 & 0.2720 & 0.1560 \\
$h_{4}$ & 0.2125 & 0.2185 & 0.2880 & 0.1700 \\
\hline
\end{tabular}

Step 6: According to Eq. (3), we can obtain $c_{1}\left(w^{\text {global }}\right)=0.0297 ; c_{2}\left(w^{\text {global }}\right)=-0.1031 ; c_{3}\left(w^{\text {global }}\right)=$ $-0.0075 ; c_{4}\left(w^{\text {global }}\right)=0.0809$.

Rank all the suppliers $h_{i}(i=1,2,3,4)$ in accordance with the scores $c_{\mathrm{i}}\left(w^{\text {global }}\right): h_{4} \succ h_{1} \succ h_{3} \succ h_{2}$, and thus the most desirable supplier is $h_{4}$

Case 2: The information about the attribute weights is completely unknown

Utilize Eq.(10) and get the attribute initial weights.

$w^{\text {initial }}=(0.28,0.48,0.13,0.11)^{T}$

Using the PSO learning method, we can obtain the finally global weights:

$w^{\text {global }}=[0.26,0.36,0.25,0.14]^{T}$

Construct the resultant weighted fuzzy soft set $\left(\tilde{F},\left(w^{\text {global }} E\right)\right)$ according to fuzzy $\operatorname{soft} \operatorname{set}(\tilde{F}, E)$, which is shown in Table 3.

According to Eq. (3), we can obtain

$c_{1}\left(w^{\text {global }}\right)=0.0137, c_{2}\left(w^{\text {global }}\right)=-0.0951, c_{3}\left(w^{\text {global }}\right)=$ $0.0077, c_{4}\left(w^{\text {global }}\right)=0.0737$.

Rank all the suppliers $h_{i}(i=1,2,3,4)$ in accordance 
with the scores $c_{\mathrm{i}}\left(w^{\text {global }}\right): h_{4} \succ h_{1} \succ h_{3} \succ h_{2}$, and thus the most desirable supplier is $h_{4}$.

Table 3. Tabular representation of the resultant weighted fuzzy soft $\operatorname{set}\left(\tilde{F},\left(w^{\text {global }} E\right)\right)$.

\begin{tabular}{lllll}
\hline$U$ & $w_{1}^{\text {global }} e_{1}$ & $w_{2}^{\text {global }} e_{2}$ & $w_{3}^{\text {global }} e_{3}$ & $w_{4}^{\text {global }} e_{4}$ \\
\hline$h_{1}$ & 0.2470 & 0.3204 & 0.2000 & 0.1246 \\
$h_{2}$ & 0.2288 & 0.3240 & 0.2000 & 0.1120 \\
$h_{3}$ & 0.2340 & 0.3348 & 0.2125 & 0.1092 \\
$h_{4}$ & 0.2210 & 0.3420 & 0.2250 & 0.1190 \\
\hline
\end{tabular}

\section{CONCLUSIONS}

In this paper, we have investigated a hybrid approach to tackle multiple criteria decision making problems with incomplete weight information in the context of fuzzy soft sets. To determine the criteria weights, the proposed approach consists of two stages. Firstly, in order to derive the initial weights, the novel decision making models determining the unknown weight vector have been developed. Secondly, we used FCM to deal with dependent and feedback effect among criteria. After completing these two stages, we can derive the global criteria weights. Next we will apply fuzzy soft sets in evaluating and selecting the most desirable alternative. Finally, a case study is presented to examine the practicality of the proposed model. The proposed hybrid method has a clear logic and has less loss of information than other literatures.

\section{ACKNOWLEDGEMENT}

This research was supported by Natural Science Foundation of China (No.71171209/G011203), the Humanities and Social Sciences Foundation of Ministry of Education of the People's Republic of China (No.10XJA630010) and Ministry of Education in China Project of Humanities and Social Sciences (13YJC630252). The authors wish to express their appreciation to the Editors of this journal and the anonymous reviewers for their constructive comments that significantly improve the quality and presentation of the paper.

\section{REFERENCES}

[1] Kahraman, C. \& Cebi, S. 2009. A new multi-attribute decision making method: Hierarchical fuzzy axiomatic design. Expert Systems with Applications. 36: 48484861.

[2] Chen, T. 2012. Multiple criteria group decision-making with generalized interval-valued fuzzy numbers based on signed distances and incomplete weights. Applied Mathematical Modelling, 36: 3029-3052.
[3] Z.S. Xu \& J. Chen. 2007. An interactive method for fuzzy multiple attributes group decision making. Inform Sciences, 177: 248-263.

[4] Z.J. Wang, K.W. Li \& W.Z. Wang. 2009. An approach to multiple attribute decision making with interval-valued intuitionistic fuzzy assessments and incomplete weights. Inform Sciences, 179: 3026-3040.

[5] J. Ye. 2010. Fuzzy decision-making method based on the weighted correlation coefficient under intuitionistic fuzzy environment. Eur J Oper Res, 205: 202-204.

[6] G.W. Wei. 2010. Extension of TOPSIS method for 2-tuple linguistic multiple attribute group decision making with incomplete weight information. Know Inf Syst, 25: 623-634.

[7] D. Molodtsov. 1999. Soft set theory-first results. Comput Math Appl, 37: 19-31.

[8] P.K. Maji \& A.R. Roy. 2002. An application of soft sets in a decision making problem. Comput Math Appl, 44: 1077-1083.

[9] M.M. Mushrif, S. Sengupta \& A.K. Ray. 2006. Texture classification using a novel, soft-set theory based classification algorithm. COMPUTER VISION-ACCV 2006, pp.246-254.

[10] Y. Zou \& Z. Xiao. 2008. Data analysis approaches of soft sets under incomplete information. Knowl-Based Syst, 21: 941-945.

[11] A.R. Roy \& P.K. Maji. 2007. A fuzzy soft set theoretic approach to decision making problems. J Comput Appl Math, 203: 412-418.

[12] Z. Kong, L.Q. Gao \& L.F. Wang. 2009. Comment on "A fuzzy soft set theoretic approach to decision making problems". J Comput Appl Math, 223: 540-542.

[13] N. Cagman \& S. Enginoglu. 2010. Soft set theory and uni-int decision making. Eur J Oper Res, 207: 848-855.

[14] F. Feng, Y.B. Jun, X. Liu \& L. Li. 2010. An adjustable approach to fuzzy soft set based decision making. $J$ Comput Appl Math, 234: 10-20.

[15] S.J. Chen \& C.L. Hwang. 1992. Fuzzy Multiple Attribute Decision Making: Method and Applications, New York: Springer-Verlag.

[16] T.L. Saaty. 1996. Decision Making with Dependence and Feedback: The Analytic Network Process: The Organization and Priorization of Complexity. Pittsburgh: RWS Publications.

[17] T.L. Saaty \& L.G. Vargas. 1998. Diagnosis with dependent symptoms: Bayes theorem and the analytic hierarchy process. Oper Res, 46: 491-502.

[18] R. Yua \& G. Tzeng. 2006. A soft computing method for multi-criteria decision making with dependence and feedback. Appl Math Computation, 180: 63-75.

[19] B. Kosko. 1996. Fuzzy Engineering, New York: Prentice Hall.

[20] Z. Xu. 2007. A method for multiple attribute decision making with incomplete weight information in linguistic setting. Knowl-Based Syst, 20: 719-725.

[21] D. Park, Y. Kwun, J. Park \& I. Park. 2009. Correlation coefficient of interval-valued intuitionistic fuzzy sets and its application to multiple attribute group decision making problems. Math Comput Model, 50: 1279-1293.

[22] S. French, R. Hartley, L.C. Thomas \& D.J. White. 1983. Multi-objective Decision Making, New York: Academic Press.

[23] Y.T. Lin et al. 2010. A novel hybrid MCDM approach for outsourcing vendor selection: A case study for a semiconductor company in Taiwan. Expert Syst Appl, 37: 4796-4804. 